\title{
O impacto da Lei Sarbanes-Oxley (SOX) na qualidade do lucro das empresas brasileiras que emitiram ADRs ${ }^{1}$
}

The impact of Sarbanes-Oxley on earnings quality of Brazilian ADR issuers

\author{
Mark Miranda de Mendonça \\ Mestre em Ciências Contábeis pela Fucape Business School \\ E-mail: mark.miranda@sicoob.com.br
}

Fábio Moraes da Costa

Professor Associado da Fucape Business School

E-mail: fabio@fucape.br

Fernando Caio Galdi

Professor Associado da Fucape Business School

E-mail: fernando.galdi@fucape.br

\section{Bruno Funchal}

Professor Associado da Fucape Business School

E-mail: bfunchal@fucape.br

Recebido em 17.03.2009 * Aceito em 20.05.2009 * $2^{\text {a }}$ versão aceita em 05.05.2010

\section{RESUMO}

Este trabalho objetiva analisar o impacto da Lei Sarbanes-Oxley (SOX) na qualidade do lucro das empresas brasileiras que emitiram ADR antes de 2002. Os escândalos contábeis de companhias como Enron e WorldCom reduziram a confiança dos investidores sobre a veracidade das demonstrações financeiras. A SOX surgiu com o propósito de proteger os investidores pela melhora na precisão e na confiabilidade na divulgação das demonstrações das empresas. Para medir a qualidade dos lucros, foram utilizados o Modelo de Basu (1997) e os modelos apresentados em Ball e Shivakumar (2005), estimados com dados em painel. Esperava-se que o aumento da regulamentação implicasse em maior grau de qualidade dos lucros nas empresas no período pós-SOX. Os resultados encontrados não são significativos nos modelos de Reversão à média das variações dos lucros e Cash Flow de Ball e Shivakumar (2005), mas os resultados do Modelo de Basu (1997) sugerem indício de aumento do conservadorismo contábil após a SOX, nas empresas brasileiras que emitiram ADR antes de 2002. Contudo, esse aumento do conservadorismo pelo Modelo de Basu (1997) também foi observado para todas as empresas brasileiras. Portanto, os resultados aqui apresentados sugerem indícios de aumento do conservadorismo brasileiro a partir de 2002, mas por outros motivos econômicos que não ocasionados pelo efeito SOX.

Palavras-chaves: Lei Sarbanes-Oxley (SOX), Conservadorismo, American Depositary Receipt (ADR).

\footnotetext{
${ }^{1}$ Artigo apresentado no $8^{\circ}$ Congresso USP de Controladoria e Contabilidade, São Paulo-SP, 2008. 


\section{ABSTRACT}

This study analyzes the impact of the Sarbanes-Oxley Act (SOX) on earnings quality of Brazilian companies qualified as ADR issuers before 2002. The accounting scandals involving firms like Enron and WorldCom reduced investors' confidence in the reliability of financial statements. SOX arose to protect investors by mandating more precise and reliable financial disclosure by firms. To measure earnings quality, we used the models by Basu (1997) and Ball and Shivakumar (2005), estimated with panel data. We expected that the enhanced legal framework would imply greater earnings quality in the period after SOX. Although the results were not significant for the conservatism and cash flow models, the results for the Basu model suggest increased conservatism after SOX in Brazilian companies that had already traded ADRs before 2002. However, this increased conservatism according to the Basu model was also observed for all Brazilian companies. Therefore, the results presented here suggest this increased conservatism by Brazilian companies after 2002 cannot be directly linked to the effects of Sarbanes-Oxley.

Keywords: Sarbanes-Oxley Act (SOX), conservatism, American Depositary Receipt (ADR).

\section{INTRODUÇÃO}

Este trabalho analisa o impacto da Lei Sarbanes-Oxley (SOX) sobre a qualidade do lucro nas demonstrações contábeis das empresas brasileiras que emitiram ADR antes de 2002.

Os escândalos contábeis de companhias americanas como Enron e a WorldCom reduziram a confiança dos investidores nas informações das demonstrações financeiras. Apesar de uma elaborada rede de governança corporativa, de uma regulamentação forte e órgãos fiscalizadores presentes (PALEPU; HEALY, 2003), essas corporações manipularam as demonstrações utilizando formas complexas de gerenciamento de resultados (LOBO; ZHOU, 2006). A assimetria informacional entre a diretoria e os acionistas e as atuações pouco rígidas do comitê de auditoria e da auditoria externa, descumprindo obrigações contratuais de proteção aos acionistas, foram primordiais para o colapso da Enron (PALEPU; HEALY, 2003).

Os escândalos demonstraram um ponto fraco no mercado de capitais americano, em que os problemas de governança corporativa poderiam emergir em muitas outras empresas, afetando, potencialmente, o mercado inteiro (PALEPU; HEALY, 2003).

É nesse contexto que a SOX surge, em 2002, para recuperar a credibilidade da informação contábil, aumentando o custo de litígio e o nível de governança corporativa. Essa lei almeja proteger os investidores e restaurar sua confiança nas demonstrações financeiras das empresas, por meio de uma maior precisão das divulgações contábeis.

Contudo, empresas estrangeiras provenientes de ambientes de fracas instituições legais nos seus países, são normalmente listadas na Bolsa de Nova Iorque, sendo que a SEC e as leis 
americanas não coíbem eventuais fraudes. Ao identificá-las, raramente têm conseguido fazer cumprir sansões legais contra qualquer empresa estrangeira listada no mercado de capitais americano (SIEGEL, 2005).

Dessa forma, se o arcabouço legal que envolve as empresas estrangeiras ao emitirem ADR não serve como barreira para evitar ou coibir fraudes, as premissas teóricas quanto ao aumento da qualidade do lucro, baseadas na redução do conflito de interesses resultado do aumento da regulamentação, não terão efeito.

Em função da repercussão dessa legislação, este estudo apresenta a seguinte questão de pesquisa: As empresas brasileiras que possuem ADRs emitidas antes de 2002 aumentaram a qualidade do lucro contábil de suas demonstrações financeiras depois da SOX?

\section{REVISÃO DA LITERATURA E REFERENCIAL TEÓRICO}

O impacto da regulamentação nas demonstrações contábeis é estudado por Watts (2003) e, de acordo com o autor, a regulamentação fornece incentivos para as empresas terem demonstrações mais conservadoras, que é uma das proxies para a qualidade dos lucros contábeis.

Dentro dessa linha de influência da regulamentação na Contabilidade, a sua relevância consiste em impactar na informação contábil, afetando, direta e indiretamente, as divulgações das empresas. Dessa forma, a regulamentação governamental, bem como as questões judiciais sob a imposição das leis, encoraja e incentiva o conservadorismo nas demonstrações financeiras das empresas (SARLO NETO et al. 2005; COSTA; COSTA, 2005; BEAVER, 1993; WATTS, 2003).

A regulamentação foi escolhida como alternativa de impacto no conservadorismo contábil, utilizando a SOX como proxy de legislação. A referida lei pode ser estudada no Brasil, pois, para negociarem nos EUA, as empresas brasileiras que emitem ADR têm que seguir a legislação norte-americana.

\subsection{Lei Sarbanes-Oxley (Sox)}

A lei norte-americana Sarbanes-Oxley teve como propósito evitar a possível fuga dos investidores financeiros, que, após os escândalos envolvendo grandes companhias como a Enron e WorldCom, estavam inseguros quanto à real qualidade das práticas de governança corporativa adotadas pelas empresas (JAIN; REZAEE, 2004).

Além dos rígidos parâmetros legais impostos às empresas com ações negociadas nas bolsas americanas, incluindo empresas estrangeiras que negociam ADR, seu conjunto de 
regras busca garantir: a criação de mecanismos confiáveis de auditoria e a criação de comitês para supervisionar suas atividades e assegurar maior independência na atuação da auditoria externa, de modo a mitigar riscos aos negócios, evitar fraudes e garantir transparência aos resultados contábeis das companhias.

A atividade de auditoria é um tema muito importante e componente-chave no ambiente da governança corporativa, que possui particular apreciação dos reguladores, profissionais, investidores, enfim, do público em geral. A área ficou ainda mais em evidência, após as falhas e os escândalos contábeis da Enron, WorldCom e outras companhias americanas (DEFOND; FRANCIS, 2005). Nesse sentido, a SOX fez mudanças radicais, principalmente nas questões relacionadas à auditoria. Algumas delas são expostas a seguir (DEFOND; FRANCIS, 2005):

- Transformou a auditoria de uma atividade autorregulada e supervisionada pela SEC, em uma atividade diretamente controlada por uma quase agência governamental: a PCAOB;

- Fez diversas mudanças com o compromisso fundamental de melhorar a independência da auditoria externa, como: a determinação da qualidade do serviço; o aumento em mais de $50 \%$ dos honorários da auditoria e os conflitos de interesses entre a administração e a empresa de auditoria;

- Estabeleceu critérios quanto à criação e à composição do comitê de auditoria, como: todas as empresas devem ter um comitê composto inteiramente por membros que são independentes da administração; o comitê deve conter no mínimo um especialista em finanças (financial expert) e, caso não possua, explicar o porquê; o comitê é responsável pela nomeação da empresa de auditoria externa; a empresa deve ter consultor externo e outros consultores que o comitê considerar necessário para cumprir as obrigações legais; o comitê deve implementar procedimentos para receber e investigar queixas de empregados sobre as práticas e políticas contábeis.

De acordo com Lobo e Zhou (2006), que analisaram o impacto da SOX no conservadorismo das empresas americanas, houve aumento no conservadorismo com o resultado assimilando mais rapidamente as más notícias do que as boas e redução do nível dos accruals discricionários no período pós-SOX. Os autores relatam outros estudos que também investigaram o impacto da SOX nas demonstrações financeiras das empresas americanas, como Cohen, Dey e Lys (2005) que, em linha com a intenção da lei de aumentar a precisão das demonstrações contábeis, encontraram redução nos accruals discricionários no período pós-SOX e Heflin e Hsu (2004) que constataram um significante declínio no uso de 
informações "Non-GAAP" para mensurar os resultados e a probabilidade dos resultados divulgados ultrapassarem as previsões dos analistas após a SOX. Por outro lado, contrário aos resultados apresentados nesses estudos, Jain e Rezaee (2004) não constataram aumento no grau do conservadorismo após a instituição da SOX.

\subsection{Conservadorismo}

Basu (1997) define conservadorismo no resultado como o reconhecimento das más notícias, mais rapidamente do que o reconhecimento das boas notícias, em relação aos fluxos de caixa futuros.

Segundo Ball et al. (2000), conservadorismo pode ser entendido como o grau do qual o período corrente do resultado contábil incorpora assimetricamente as perdas e ganhos econômicos. Lopes (2001), também, entende que o conservadorismo é o reconhecimento assimétrico entre despesas e passivos, receita e ativos, e complementa relatando sobre a importância do conservadorismo ao fornecer informações mais confiáveis aos investidores por intermédio de demonstrações não excessivamente otimistas.

Segundo Ball et al. (2000), o incentivo para a utilização do conservadorismo pode estar relacionado ao modelo legal do país. Em modelos common-law, com mercado de capitais desenvolvido e controle acionário descentralizado, o grau de conservadorismo é maior do que em países com modelo code-law, com mercado menos desenvolvido e controle acionário centralizado. Contudo, constataram em todos os países que há reconhecimento das más notícias mais rapidamente do que as boas notícias, independente do modelo legal do país.

Dentro dessa linha que envolve o conservadorismo e o mercado de capitais, autores como Ball et al. (2000), Watts (2003), Lubberink e Huijgen (2000), Pope e Walker (1999) e Penman e Zhang (2002), desenvolveram testes empíricos sobre a relação entre o conservadorismo contábil nos lucros e o mercado acionário. Os autores, em sua maioria, defendem a utilização do conservadorismo no lucro contábil, com exceção do trabalho de Penman e Zhang (2002) no qual demonstram que a utilização do conservadorismo pode gerar lucro de baixa qualidade.

Na América do Sul, seguindo a literatura sobre o tema, os resultados do estudo de Costa e Costa (2005) evidenciaram baixa relação entre o lucro corrente e o retorno corrente nos cinco países analisados.

A literatura sobre o conservadorismo no Brasil desenvolveu-se consideravelmente nos últimos anos. Costa, Almeida e Scalzer (2006) encontraram evidências de que o grau de conservadorismo nas empresas que possuem níveis diferenciados de governança corporativa 
(listadas no Nível 1, Nível 2 ou Novo Mercado) é maior do que as demais empresas listadas na BOVESPA que não possuem tais níveis. Santos (2006) avalia a existência de diferenças no conservadorismo de empresas brasileiras que possuem ADRs e, portanto, publicam seus resultados em US-GAAP e BR-GAAP. Seus resultados indicam que não há diferenças significativas no conservadorismo dessas empresas em BR-GAAP e em US-GAAP. Paulo, Antunes e Formigoni (2006) avaliam a distinção da prática de conservadorismo entre empresas abertas e fechadas e encontram evidencias de Maior conservadorismo apresentado nas empresas abertas. Coelho e Lima (2007) investigam a presença de conservadorismo nos resultados das sociedades por ação no período de 1995 a 2004 e seus resultados indicam que as empresas avaliadas não praticam o conservadorismo condicional, embora se constate reversão dos componentes transitórios do lucro em maior magnitude no caso de variações negativas do lucro antecedente.

Coelho, Cia e Lima (2008) testam o nível de conservadorismo condicional das firmas brasileiras listadas no mercado americano em relação àquelas não emissoras de ADR e quais as diferenças de tal atributo nas demonstrações contábeis das emissoras de ADR. Os autores encontram indícios de que diferença de princípios contábeis não implica diferenças nos graus de atributos aceitos como indicativos de qualidade nos lucros publicados. A presente pesquisa vem a complementar as evidências já encontradas no mercado brasileiro e agrega ao que considera modelos diferentes para a avaliação da qualidade do lucro contábil antes e depois da SOX.

\subsubsection{Análise Complementar da qualidade dos lucros contábeis}

De acordo com Watts (2003a), o gerenciamento de resultados pode explicar o conservadorismo contábil nas empresas, desde que seja analisado em conjunto com uma das principais alternativas (contratos, questões judiciais, impostos e regulamentação) de impacto. Sendo que essa alternativa complementar não pode, individualmente, explicar a máxima do conservadorismo contábil que é o reconhecimento assimétrico das perdas e ganhos no resultado.

Utiliza-se, neste trabalho, o gerenciamento de resultados como alternativa complementar de análise do conservadorismo. Comparando as empresas nos períodos pré e pós-SOX, as que demonstrarem menor nível de accruals discricionários estarão gerenciando menos os resultados, indicando maior conservadorismo nas suas demonstrações contábeis.

Nesse contexto, Watts (2003a) afirma que o gerenciamento de resultados, mediante a mensuração do grau do accruals discricionários - a porção dos accruals que sofrem 
intervenção dos gestores - pode ser utilizado como uma das medidas para determinar o grau do conservadorismo.

Observe-se que os accruals discricionários possuem um importante papel informacional, sendo que os métodos para detectar e analisar o gerenciamento de resultados, nas empresas, se baseiam no uso desses accruals pelos gestores, uma vez que eles conduzem o resultado que mais lhes interessa ou à empresa. Modelos como os de Healy, Jones, Jones Modificado e Kang e Silvaramakrishnan (KS), têm sido utilizados frequentemente pelos pesquisadores (COELHO; LOPES, 2005; MARTINEZ, 2001). Neste trabalho, para determinar o nível dos accruals discricionários, utilizou-se outra proxy de conservadorismo baseado em accruals, o Cash Flow Model (Modelo de Fluxo de Caixa), em linha com os estudos de Ball e Shivakumar (2005).

\subsection{American Depositary Receipt - ADR}

De acordo com Downes e Goodman (1993), o Recibo de Depósito Americano ou ADR (American Depositary Receipt) é o recibo de ações de empresas sediadas fora dos EUA, custodiado por um banco americano e outorgando ao acionista o direito sobre todos os dividendos e ganhos de capital. Em vez de comprar ações de companhias estrangeiras nos mercados externos, os americanos podem comprá-las nos EUA sob forma de um ADR.

Dentro desse contexto, as empresas estrangeiras utilizam ADR como substituto parcial da fraca legislação em seus países e também para ajudar a atrair mais capital por meio de financiamento externo (COFFEE, 1999). Assim, Coffee (1999), utilizando a teoria da agência, afirma que a combinação da supervisão da SEC e sanções das leis americanas podem proteger os acionistas minoritários, coibindo efetivamente possíveis fraudes de empresas estrangeiras listadas no mercado de capitais americano, minimizando, assim, o custo de agência.

Em relação ao nível de governança corporativa, Lubberink e Huijgen (2006) afirmam que os administradores só submetem suas empresas a um alto nível de governança via ADR, se as oportunidades de crescimento forem financiadas com o uso de recursos externos. Segundo eles, isso ocorre principalmente em países com características code-law.

O aumento nos mecanismos de governança e no custo de litígio, objetivando reduzir o conflito de interesses entre os agentes e provocar o cumprimento das obrigações contratuais, assuntos propostos pela SOX, pode não impactar no aumento do conservadorismo contábil nas empresas que emitem ADR. Isso, de fato, tende a ocorrer, caso a SEC e o arcabouço legal americano realmente não forem eficientes na coerção e punição das fraudes, bem como não reunirem condições de proteger os interesses dos investidores. 


\section{HIPÓTESE DE TRABALHO}

Para responder à questão de pesquisa, serão utilizados os modelos de conservadorismo de Basu (1997) e de Ball e Shivakumar (2005), e o modelo Cash Flow de gerenciamento de resultados de Ball e Shivakumar (2005). Recentemente, a literatura trata esses modelos como proxies da qualidade do lucro.

Esses modelos estão em linha com Watts (2003b), que aponta o reconhecimento assimétrico das más e boas notícias e o nível de accruals discricionários como medidas de conservadorismo das demonstrações contábeis. Dessa forma, a hipótese de trabalho será a seguinte:

$\mathrm{H}_{1}$ : A qualidade do lucro contábil aumentou depois da SOX nas demonstrações contábeis das empresas brasileiras que emitiram ADR antes de 2002.

\section{METODOLOGIA}

Nesse capítulo, descreve-se a metodologia de cálculo de cada modelo, para medir o conservadorismo e o nível de accruals discricionários. Todas as regressões dos modelos citados foram estimadas em painel no Stata 9.0, analisando-se os pressupostos das regressões e utilizando-se recursos de efeito fixo e erro padrão robusto. Devido a questões estruturais dos modelos utilizados, com exceção do pressuposto de multicolinearidade, todos os outros pressupostos estatísticos foram satisfeitos.

A análise é dividida em dois momentos: no primeiro, analisam-se, apenas, as empresas com ADR emitidas antes de 2002 e introduz-se, nos três modelos, uma dummy "ANO" para captar o efeito do período 2002 a 2006 (pós-SOX). O coeficiente dessa dummy expressaria, caso significante, a variação do conservadorismo apenas nas empresas com ADR. Contudo, ainda não se poderia afirmar que se trata do efeito SOX, pois esse aumento pode decorrer de algum fenômeno ocorrido no mercado brasileiro no período de 2002 a 2006.

Dessa forma, no segundo momento, tenta-se isolar o efeito SOX dos demais efeitos do mercado brasileiro. Nessa etapa, todas as empresas brasileiras são estudadas e se introduz uma nova dummy "ADR" multiplicando a dummy "ANO", para captar o efeito adicional ocorrido nas empresas com ADR. Entende-se que o coeficiente do termo "ADR*ANO" expressaria, se significante, indícios do impacto da SOX no conservadorismo contábil das empresas que emitiram ADR antes de 2002. 
Assim, o coeficiente da dummy "ANO" mensuraria o efeito "economia brasileira" e o coeficiente da dummy "ADR" captaria o efeito "estar no mercado americano". Essa mensuração adicional seria, então, um indicativo de efeito "SOX". Se o coeficiente da multiplicação entre as dummies for significativo, haverá indícios do aumento do conservadorismo pela SOX, caso contrário, rejeita-se a hipótese do trabalho.

\subsection{Empresas que emitiram ADR antes de 2002}

Pretende-se apurar o efeito da SOX, a partir do ano de sua promulgação, no conservadorismo das empresas brasileiras que emitiram ADR antes de 2002.

Contudo, ressalte-se que qualquer outro fenômeno que esteja ocorrendo a partir de 2002 no mercado brasileiro poderá ser captado pela dummy “ANO”. Assim, o teste descrito no tópico 4.2 faz-se necessário.

\subsubsection{Modelo de Basu (1997)}

O modelo de Basu (1997) é utilizado para mensurar o conservadorismo contábil nas demonstrações financeiras das empresas. Esse método avalia se os resultados incorporam más notícias mais rapidamente do que boas notícias, conforme demonstrado na Equação 1:

$\mathrm{LPA}_{\mathrm{i}, \mathrm{t}} / \mathrm{P}_{\mathrm{i}, \mathrm{t}-1}=\alpha_{0}+\alpha_{1} \mathrm{D}_{\mathrm{i}, \mathrm{t}}+\alpha_{2} \mathrm{R}_{\mathrm{i}, \mathrm{t}}+\alpha_{3} \mathrm{D}_{\mathrm{it}} * \mathrm{R}_{\mathrm{i}, \mathrm{t}}+\alpha_{4} \mathrm{~A}_{\mathrm{i}, \mathrm{t}} * \mathrm{D}_{\mathrm{i}, \mathrm{t}} * \mathrm{R}_{\mathrm{i}, \mathrm{t}}+\varepsilon_{\mathrm{i}, \mathrm{t}}$

em que:

$\mathrm{LPA}_{\mathrm{i}, \mathrm{t}}=$ Lucro Por Ação (lucro/prejuízo) da empresa $i$ no período $t$;

$\mathrm{D}_{\mathrm{i}, \mathrm{t}}=$ Variável dummy assume valor 1 se o retorno econômico for negativo e valor 0 nos demais casos;

$\mathrm{R}_{\mathrm{i}, \mathrm{t}}=$ Retorno econômico da ação da empresa $i$ no período $t$ (entre abril do ano $t \mathrm{e}$ abril do ano $t+1)$;

$\mathrm{A}_{\mathrm{i}, \mathrm{t}}=$ Variável dummy (ANO) que assume valor 1 para as observações a partir do ano de 2002 (promulgação da SOX) e valor 0 nos demais casos;

$\mathrm{P}_{\mathrm{i}, \mathrm{t}-1}=$ Preço da ação da empresa $i$ que contém a precificação no ano $t-1$ (abril do ano $t$ subsequente);

$\alpha_{2}=$ Reflete a oportunidade do lucro contábil;

$\alpha_{1}, \alpha_{3}$ e $\alpha_{4}=$ Refletem o reconhecimento assimétrico do retorno econômico, as boas e más notícias, pelo lucro contábil;

$\varepsilon_{\mathrm{it}}=$ Termo de erro estatístico.

A análise do coeficiente $\alpha_{0}$ não se faz relevante, por não conter significado teórico econômico. A utilização de uma variável dummy permite verificar se o lucro contábil é mais 
sensível aos resultados negativos que aos positivos, o seu coeficiente $\alpha_{1}$ mensura a defasagem temporal. A intensidade dessa defasagem é mensurada pelo coeficiente $\alpha_{3}$. Esses dois coeficientes refletem o reconhecimento assimétrico do retorno econômico pelo lucro contábil. O coeficiente $\alpha_{2}$ reflete a oportunidade do lucro contábil, ou seja, o reconhecimento do retorno econômico pelo lucro contábil.

Se houver conservadorismo no reconhecimento do retorno econômico pelo lucro, o $\alpha_{2}$ deverá incorporar mais significativamente o retorno econômico negativo que o positivo, sendo esperado que o coeficiente $\alpha_{3}$ seja positivo. Maior diferença entre os coeficientes $\alpha_{3} \mathrm{e}$ $\alpha_{2}$ indica maior grau de conservadorismo contábil, esperando-se sinal negativo e significativo para $\alpha_{1}$ e positivo e significativo para $\alpha_{3}$.

Note-se que a introdução da variável " $\mathrm{A}_{\mathrm{i}, \mathrm{t}}$ " faz com que o coeficiente $\alpha_{4}$ se configure no principal instrumento para a análise do conservadorismo no presente trabalho. $\mathrm{O} \alpha_{4}$ mensura a alteração do conservadorismo a partir de 2002, ano de promulgação da SOX, ou seja, a variação da intensidade de defasagem temporal entre o reconhecimento contábil de boas e más notícias já reconhecidas pelo mercado. Espera-se sinal positivo e significativo para o coeficiente $\alpha_{4}$.

\subsubsection{Modelo de Ball e Shivakumar (2005)}

Optou-se, também, por adotar outro modelo, de Ball e Shivakumar (2005), de agora em diante denominado BS, objetivando a complementação do estudo de mensuração do grau de conservadorismo contábil das empresas.

Esse modelo utiliza como variáveis a variação do lucro e permite identificar o nível de conservadorismo por meio da reversão dos resultados (ganhos ou perdas) contábeis (BALL; SHIVAKUMAR, 2005). O modelo utilizado por Ball e Shivakumar (2005) utiliza a variação do lucro líquido dividida pelo valor do ativo total no início do período. O modelo utilizado nesse estudo adapta o modelo original e considera a variação do lucro por ação, ao invés do lucro líquido, por diminuir o possível viés de seleção no caso da diminuição da amostra decorrente do escalonamento ${ }^{2}$. Efeitos de possíveis viés de seleção e suas influências nos resultados são discutidos em Durtschi e Easton (2009). O modelo BS adaptado mede a assimetria de reconhecimento entre as receitas (boa notícia) e as despesas (má notícia), conforme a Equação (2) abaixo:

\footnotetext{
${ }^{2}$ No período da amostra, diversas empresas possuem um valor significativamente pequeno de Ativo, o que pode influenciar o resultado em análise: Em 2002, 36 empresas possuíam ativos inferiores a $\mathrm{R} \$ 30$ milhões de Reais. Em 2003, esse número foi de 29, em 2004 de 30, em 2005 de 30 e em 2006 de 32 empresas. Nesse sentido, optou-se por trabalhar com o Lucro por Ação por não ser impactado por esse efeito.
} 
VLPA $_{i, t}=\alpha_{0}+\alpha_{1} D_{i, t} * \operatorname{VLPA}_{i, t-1}+\alpha_{2} \operatorname{VLPA}_{\mathrm{i}, \mathrm{t}-1}+\alpha_{3} \operatorname{VLPA}_{\mathrm{i}, \mathrm{t}} * \mathrm{D}_{\mathrm{i}, \mathrm{t}} * \operatorname{VLPA}_{\mathrm{i}, \mathrm{t}}+\alpha_{4} \mathrm{~A}_{\mathrm{i}, \mathrm{t}} * \mathrm{VLPA}_{\mathrm{i}, \mathrm{t} 1}$

$+\alpha_{5} \mathrm{~A}_{\mathrm{i}, \mathrm{t}} * \mathrm{VLPA}_{\mathrm{i}, \mathrm{t}-1} * \mathrm{D}_{\mathrm{i}, \mathrm{t}} * \mathrm{VLPA}_{\mathrm{i}, \mathrm{t}}+\varepsilon_{\mathrm{i}, \mathrm{t}}$

em que:

VLPA $_{\mathrm{i}, \mathrm{t}}=$ Variação do Lucro Por Ação (contábil) da empresa $i$, do ano t-1 para o ano t; VLPA $_{\mathrm{i}, \mathrm{t}-1}=$ Variação do Lucro Por Ação (contábil) da empresa $i$, do ano t-2 para o ano $\mathrm{t}-1$;

$\mathrm{D}_{\mathrm{i}, \mathrm{t}} * \mathrm{VLPA}_{\mathrm{i}, \mathrm{t}-1}=$ Variável dummy para indicar se existe variação negativa do lucro por ação no ano t-1 para o ano t, assumindo valor 1 se $\mathrm{VLPA}_{\mathrm{t}-1}<0$, e 0 nos demais casos; $\mathrm{A}_{\mathrm{i}, \mathrm{t}}=$ Variável dummy (ANO) que assume valor 1 para as observações a partir do ano de 2002 (promulgação da SOX) e valor 0 nos demais casos;

$\alpha_{0}, \alpha_{1}, \alpha_{2}, \alpha_{3}, \alpha_{4}$ e $\alpha_{5}=$ estimadores dos coeficientes;

$\varepsilon_{\mathrm{i}, \mathrm{t}}=$ termo de erro estatístico.

A análise dos parâmetros $\alpha_{0}$ e $\alpha_{1}$ não é relevante, pois eles não trazem significado teórico econômico, não existindo uma predição para esses coeficientes.

Devido à diferença do reconhecimento dos ganhos até o momento em que o fluxo de caixa é realizado, em que os resultados positivos se tornam um componente persistente do lucro contábil, tendendo a não ser revertido, espera-se que o coeficiente $\alpha_{2}$ seja igual a zero (não significativo). No caso desse reconhecimento não ser postergado, os ganhos passam a ser um componente transitório do resultado e nos períodos seguintes tendem a ser revertidos, implicando o coeficiente $\alpha_{2}$ menor que zero $\left(\alpha_{2}<0\right)$.

O coeficiente $\alpha_{3}$ estima a ocorrência de reversão de resultados contábeis negativos (variações negativas do resultado). O reconhecimento mais oportuno das perdas do que dos ganhos, resulta em decréscimos transitórios do resultado, devendo ser revertidos nos períodos subsequentes. Assim, espera-se que o coeficiente $\alpha_{3}$ seja estatisticamente negativo $\left(\alpha_{3}<0\right)$.

A introdução da variável " $A_{i, t}$ " faz com que os coeficientes $\alpha_{4}$ e $\alpha_{5}$ mensurem a alteração da frequência da reversão de resultados contábeis positivos e negativos a partir de 2002, ano da promulgação da SOX.

Espera-se que os componentes positivos do resultado não sejam revertidos após a promulgação da SOX, de modo que o sinal esperado para $\alpha_{4}$ é nulo (estatisticamente não significativo). Contudo, espera-se que a frequência da reversão de resultados negativos se eleve a partir de 2002, de modo que o sinal esperado para $\alpha_{5}$ seja estatisticamente negativo $\left(\alpha_{5}<0\right)$. 


\subsubsection{Cash Flow Model (BALL; SHIVAKUMAR, 2005)}

Se a administração da empresa é mais conservadora seguindo a SOX, ela tenderá a usar menos accruals discricionários, ou seja, espera-se que o nível de accruals seja menor seguindo a SOX (regulamentação) do que no período pré-SOX.

Para analisar o nível de accruals, utilizou-se o Cash Flow Model, proxy de conservadorismo baseado em accruals, de acordo com o trabalho de Ball e Shivakumar (2005), conforme demonstrado na Equação 5. O ponto de partida para medir o accrual discricionário (AD) é o cálculo do accrual total, conforme Equação 3:

$$
\mathrm{ACT}_{\mathrm{i}, \mathrm{t}}=\mathrm{LL}_{\mathrm{i}, \mathrm{t}}-\mathrm{FCO}_{\mathrm{i}, \mathrm{t}}
$$

em que:

$\mathrm{ACT}_{\mathrm{i}, \mathrm{t}}=$ Accruals Totais da empresa $i$ no período $\mathrm{t}$;

$\mathrm{LL}_{\mathrm{i}, \mathrm{t}}=$ Lucro Líquido da empresa $i$ no período $\mathrm{t}$;

$\mathrm{FC}_{\mathrm{i}, \mathrm{t}}=$ Fluxo de Caixa Operacional da empresa $i$ no período $\mathrm{t}$.

Após a mensuração dos accruals totais, calculam-se os accruals não discricionários por meio do Cash Flow Model (BALL; SHIVAKUMAR, 2005). Segundo Ball e Shivakumar (2005, p. 15), espera-se $\alpha_{1}<0$, em que a variação das operações de fluxo de caixa é a única variável explicativa. Os autores afirmam, ainda, que essa previsão assume a correlação negativa em função da redução do comportamento dos accruals.

$\mathrm{AND}_{\mathrm{i}, \mathrm{t}}=\alpha_{1} \mathrm{FCO}_{\mathrm{i}, \mathrm{t}}$

em que:

$\mathrm{AND}_{\mathrm{i}, \mathrm{t}}=$ Accruals não discricionários da empresa $i$ no período t;

$\alpha_{1}=$ coeficiente estimado pela seguinte regressão:

$\mathrm{ACT}_{\mathrm{i}, \mathrm{t}}=\alpha_{0}+\alpha_{1} \mathrm{FCO}_{\mathrm{i}, \mathrm{t}}+\alpha_{2} \mathrm{~A}_{\mathrm{i}, \mathrm{t}}+\varepsilon_{\mathrm{i}, \mathrm{t}}$

em que:

$\mathrm{A}_{\mathrm{i}, \mathrm{t}}=$ Variável dummy (ANO) que assume valor 1 para as observações a partir do ano de 2002 (promulgação da SOX) e valor 0 nos demais casos;

$\alpha_{0}, \alpha_{1}$ e $\alpha_{2}=$ estimadores dos coeficientes;

$\varepsilon_{\mathrm{t}}=$ termo de erro estatístico.

Com a introdução da variável " $\mathrm{A}_{\mathrm{i}, \mathrm{t}}$ ", espera-se que o seu coeficiente $\alpha_{2}$ seja estatisticamente negativo, em função da redução do comportamento dos accruals em decorrência da redução dos resíduos (accruals discricionários). 


\subsection{Todas as empresas brasileiras}

Nesse tópico, pretende-se avaliar se a alteração no conservadorismo das empresas com ADR emitidas antes de 2002 ocorreu pelo efeito SOX ou por outros motivos econômicos ocorridos no mercado brasileiro no período de 2002 a 2006. Acrescenta-se a essas empresas, as empresas que não emitiram ADR e que compõem a amostragem final dos dados.

\subsubsection{Modelo de Basu (1997)}

Na Equação (1), referente ao modelo de Basu (1997), acrescenta-se a variável "ADR $\mathrm{i}, \mathrm{t}$ ", conforme a Equação (6), a seguir:

$\mathrm{LPA}_{\mathrm{i}, \mathrm{t}} / \mathrm{P}_{\mathrm{i}, \mathrm{t} 1}=\alpha_{0}+\alpha_{1} \mathrm{D}_{\mathrm{i}, \mathrm{t}}+\alpha_{2} \mathrm{R}_{\mathrm{i}, \mathrm{t}}+\alpha_{3} \mathrm{D}_{\mathrm{i}, \mathrm{t}} * \mathrm{R}_{\mathrm{i}, \mathrm{t}}+\alpha_{4} \mathrm{~A}_{\mathrm{i}, \mathrm{t}} * \mathrm{D}_{\mathrm{i}, \mathrm{t}} * \mathrm{R}_{\mathrm{i}, \mathrm{t}}+\alpha_{5} \mathrm{ADR}_{\mathrm{i}, \mathrm{t}} * \mathrm{~A}_{\mathrm{i}, \mathrm{t}} * \mathrm{D}_{\mathrm{i}, \mathrm{t}} * \mathrm{R}_{\mathrm{i}, \mathrm{t}}+\varepsilon_{\mathrm{i}, \mathrm{t}}$

em que:

$\mathrm{ADR}_{\mathrm{i}, \mathrm{t}}=$ Variável dummy (ADR) que assume valor 1 para as empresas que emitiram ADR e valor 0 nos demais casos.

Em relação ao impacto da emissão de ADR sobre o conservadorismo mensurado pelo modelo de Basu (1997), espera-se que o coeficiente $\alpha_{5}$ seja positivo e significativo, o que indicaria que a velocidade de transmissão das informações negativas pela contabilidade aumenta a partir de 2002 (promulgação da SOX).

\subsubsection{Modelo de BS (2005)}

Na Equação (2), referente ao modelo de BS (2005), acrescenta-se a variável "ADR, ,t, conforme a Equação (7), a seguir:

$$
\begin{aligned}
& \operatorname{VLPA}_{\mathrm{i}, \mathrm{t}}=\alpha_{0}+\alpha_{1} \mathrm{D}_{\mathrm{i}, \mathrm{t}} * \mathrm{VLPA}_{\mathrm{i}, \mathrm{t}}+\alpha_{2} \mathrm{VLPA}_{\mathrm{i}, \mathrm{t}-1}+\alpha_{3} \mathrm{VLPA}_{\mathrm{i}, \mathrm{t}-1} * \mathrm{D}_{\mathrm{i}, \mathrm{t}} * \mathrm{VLPA}_{\mathrm{i}, \mathrm{t}}+\alpha_{4} \mathrm{~A}_{\mathrm{i}, \mathrm{t}} * \mathrm{VLPA}_{\mathrm{i}, \mathrm{t}-1}+ \\
& \alpha_{5} \mathrm{~A}_{\mathrm{i},} * \mathrm{VLPA}_{\mathrm{i}, \mathrm{t1}} * \mathrm{D}_{\mathrm{i}, \mathrm{t}} * \mathrm{VLPA}_{\mathrm{i}, \mathrm{t}}+\alpha_{6} \mathrm{ADR}_{\mathrm{i}, \mathrm{t}} * \mathrm{~A}_{\mathrm{i}, \mathrm{t}} * \mathrm{VLPA}_{\mathrm{i}, \mathrm{t}}+ \\
& \alpha_{7} \mathrm{ADR}_{\mathrm{i}, \mathrm{t}} * \mathrm{~A}_{\mathrm{i}, \mathrm{t}} * \mathrm{VLPA}_{\mathrm{i}, \mathrm{t}} * \mathrm{D}_{\mathrm{i}, \mathrm{t}} * \mathrm{VLPA}_{\mathrm{i}, \mathrm{t}}+\varepsilon_{\mathrm{i}, \mathrm{t}} \\
& \text { em que: }
\end{aligned}
$$

$\mathrm{ADR}_{\mathrm{i}, \mathrm{t}}=$ Variável dummy (ADR) que assume valor 1 para as empresas que emitiram ADR e valor 0 nos demais casos.

Em relação ao impacto da emissão de ADR sobre os resultados obtidos pelo modelo de BS (2005), espera-se que o coeficiente $\alpha_{6}$ seja insignificante e que o $\alpha_{7}$ seja positivo e significativo, o que indicaria que a frequência da reversão das informações contábeis negativas se eleva especificamente para as empresas que emitiram ADR a partir de 2002.

\subsubsection{Cash Flow Model (BALL e SHIVAKUMAR, 2005)}

Na Equação (5), referente ao modelo de Cash Flow Model (BALL; SHIVAKUMAR,

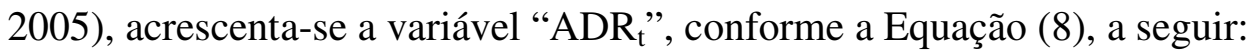


$\mathrm{ACT}_{\mathrm{i}, \mathrm{t}}=\alpha_{0}+\alpha_{1} \mathrm{FCO}_{\mathrm{i}, \mathrm{t}}+\alpha_{2} \mathrm{~A}_{\mathrm{i}, \mathrm{t}}+\alpha_{3} \mathrm{ADR}_{\mathrm{i}, \mathrm{t}} * \mathrm{~A}_{\mathrm{i}, \mathrm{t}}+\varepsilon_{\mathrm{i}, \mathrm{t}}$

em que:

$\mathrm{ADR}_{\mathrm{i}, \mathrm{t}}=$ Variável dummy (ADR) que assume valor 1 para as empresas que emitiram ADR e valor 0 nos demais casos.

Em relação ao impacto da emissão de ADR sobre o nível de accruals mensurado pelo Cash Flow Model (BALL; SHIVAKUMAR, 2005), espera-se que o coeficiente $\alpha_{6}$, da Equação 7, seja negativo e significativo, o que indicaria redução no nível de accruals discricionários especificamente para as empresas que emitiram ADR a partir de 2002.

\section{SELEÇÃO DA AMOSTRA E TRATAMENTOS DOS DADOS}

A amostra deste trabalho foi extraída do banco de dados da Economatica (valores nominais do final do ano, não corrigidos pela inflação) e se referem ao período de 1996 a 2006. Esse período foi escolhido devido à relativa estabilidade econômica brasileira após o Plano Real.

Coletaram-se os dados nominais sobre as variáveis dos três modelos de 31 de dezembro no período entre 1994 e 2006, das demonstrações consolidadas, quando disponíveis. Coletou-se, também, o preço de fechamento das ações nominal de 30 de abril no período de 1995 e 2006, utilizando-se tolerância de 15 dias.

Inicialmente, obtiveram-se 8.125 observações, referentes às 625 empresas disponíveis na Economática e aos 13 anos coletados (1994 a 2006). Em seguida, excluiram-se as 1.250 entradas referentes aos anos de 1994 e 1995, por não ser possível calcular a variável "VLPA 1" da Equação (2) para esses anos sem as informações de dezembro de 1992 e 1993, respectivamente. Excluiram-se, também, 4.905 observações com dados ausentes, não disponíveis e/ou extremos, referentes às variáveis dos três modelos, conforme a Tabela 1.

Tabela 1 - Coleta de dados - procedimento inicial de amostragem

\begin{tabular}{l|r}
\hline Anos coletados: 1994 a 2006 & 13 \\
Empresas disponíveis na Economática & 625 \\
\hline (=) Entradas totais & 8.125 \\
(-) Exclusão dos anos de 1994 e 1995 & $(1.250)$ \\
\hline (=) Dados potenciais & 6.875 \\
(-) Dados ausentes & $(4.905)$ \\
\hline (=) Entradas válidas & 1.970 \\
\hline
\end{tabular}


As 1.970 observações válidas referentes a 351 empresas foram reduzidas após a exclusão de 1.310 observações relativas a 291 empresas, cujas informações do período de 1996 a 2006 não estavam completas (descontínuas) e, assim, obtiveram-se 660 observações de empresas com informações contínuas no período estudado.

A Tabela 2 explicita que as 660 observações referentes a 60 empresas foram divididas em empresas que possuem ADR emitidas antes de 2002 (9 empresas e 99 observações) e por empresas que não possuem ADR (51 empresas e 561 observações).

Tabela 2 - Coleta de dados - procedimento final de amostragem

\begin{tabular}{c|c|c}
\hline Amostragem & Observações & Empresas \\
\hline Empresas com ADR emitidas antes de 2002 & 99 & 9 \\
Empresas sem ADR emitidas & 561 & 51 \\
\hline Amostra final & 660 & 60 \\
\hline
\end{tabular}

\section{ANÁLISE DOS RESULTADOS}

Nessa seção são apresentados os resultados dos testes com os modelos Basu (1997) e BS (2005), para análise do conservadorismo contábil e Cash Flow Model (2005), para a identificação do nível de accruals discricionários, todos estimados em painel.

Os resultados estimados pelos modelos estão divididos em: empresas que emitiram ADR antes de 2002 e todas as empresas brasileiras.

\subsection{Análise dos resultados das empresas que emitiram ADR antes de 2002}

Nesse capítulo, serão apresentados os resultados dos testes com os modelos Basu (1997) e BS (2005), para análise do conservadorismo contábil e Cash Flow Model (2005), para a identificação do nível de accruals discricionários, todos estimados em painel.

Os resultados estimados pelos modelos estão divididos em: empresas que emitiram ADR antes de 2002 e todas as empresas brasileiras.

\subsubsection{Análise dos resultados do Modelo de Basu (1997)}

Apresentam-se na Tabela 3, os resultados das regressões estimadas em painel pelo Modelo de Basu (1997) para as empresas que emitiram ADR antes de 2002. 
Tabela 3 - Resultados da regressão - Amostra só com empresas com ADR - Modelo de Basu (1997)

Equação (6) - $\mathrm{LPA}_{\mathrm{i}, \mathrm{t}} / \mathrm{P}_{\mathrm{i}, \mathrm{t}}=\alpha_{0}+\alpha_{1} \mathrm{D}_{\mathrm{i}, \mathrm{t}}+\alpha_{2} \mathrm{R}_{\mathrm{i}, \mathrm{t}}+\alpha_{3} \mathrm{D}_{\mathrm{i}, \mathrm{t}} * \mathrm{R}_{\mathrm{i}, \mathrm{t}}+\alpha_{4} \mathrm{~A}_{\mathrm{i}, \mathrm{t}} * \mathrm{D}_{\mathrm{i}, \mathrm{t}} * \mathrm{R}_{\mathrm{i}, \mathrm{t}}+\varepsilon_{\mathrm{i}, \mathrm{t}}$

\begin{tabular}{c|c|c|c|c}
\hline $\begin{array}{c}\text { Período: 1996 a 2006 } \\
\mathbf{N}^{\circ} \text { de observações: 99 } \\
\mathbf{N}^{\circ} \text { de empresas: 9 }\end{array}$ & Coeficiente & P-value & Erro Padrão Robusto & Teste t \\
\hline$\alpha_{1} \mathrm{D}_{\mathrm{i}, \mathrm{t}}$ & 0,008 & 0,865 & 0,047 & 0,17 \\
\hline$\alpha_{2} \mathrm{R}_{\mathrm{i}, \mathrm{t}}$ & 0,176 & $0,092^{*}$ & 0,103 & 1,70 \\
\hline$\alpha_{3} \mathrm{D}_{\mathrm{i}, \mathrm{t}} \mathrm{R}_{\mathrm{i}, \mathrm{t}}$ & 0,389 & 0,174 & 0,284 & 1,37 \\
\hline$\alpha_{4} \mathrm{~A}_{\mathrm{t}}{ }^{*} \mathrm{D}_{\mathrm{i}, \mathrm{t}} * \mathrm{R}_{\mathrm{i}, \mathrm{t}}$ & 0,047 & $0,039^{* *}$ & 0,022 & 2,10 \\
\hline
\end{tabular}

Notas: **Significância estatística de 5\%; * Significância estatística de $10 \%$.

Observe-se que as variáveis " $D_{i, t}$ " e " $D_{i, t} * R_{i, t}$ " não revelaram coeficientes $\alpha_{1}$ e $\alpha_{3}$ significativos, respectivamente, mesmo ao nível de significância de 10\%. Utilizando-se o modelo de Basu (1997) não se pode inferir que a informação contábil das empresas com ADR seja conservadora. Por outro lado, o conservadorismo mensurado pelo modelo de Basu (1997) apresentou aumento a partir de 2002 (ano de promulgação da SOX), vez que a variável " $\mathrm{A}_{\mathrm{i}, \mathrm{t}} * \mathrm{D}_{\mathrm{i}, \mathrm{t}} * \mathrm{R}_{\mathrm{i}, \mathrm{t}}$ " apresentou coeficiente significativo ao nível de $5 \%$.

Dessa forma, permite-se dizer que há indícios de que a informação contábil das empresas com ADR se tornou mais conservadora a partir do ano de 2002, mas ainda não se pode afirmar que há indícios de que a SOX contribuiu para esses resultados.

\subsubsection{Análise dos Resultados do Modelo adaptado de BS (2005)}

Apresentam-se, na Tabela 4, os resultados das regressões estimadas em painel pelo Modelo de BS (2005) para as empresas que emitiram ADR antes de 2002.

Tabela 4 - Resultados da regressão - Amostra só com empresas com ADR - Modelo de BS (2005)

Equação (2) - VLPA $A_{i, t}=\alpha_{0}+\alpha_{1} D_{i, t} * \operatorname{VLPA}_{i, t-1}+\alpha_{2} \operatorname{VLPA}_{\mathrm{i}, \mathrm{t}-1}+\alpha_{3} \operatorname{VLPA}_{\mathrm{i}, \mathrm{t}-1} * \mathrm{D}_{\mathrm{i}, \mathrm{t}} * \mathrm{VLPA}_{\mathrm{i}, \mathrm{t}}+\alpha_{4} \mathrm{~A}_{\mathrm{i}, \mathrm{t}} * \mathrm{VLPA}_{\mathrm{i}, \mathrm{t}-1}+$

$$
\alpha_{5} \mathrm{~A}_{\mathrm{i}, \mathrm{t}} * \mathrm{VLPA}_{\mathrm{i}, \mathrm{t}-\mathrm{1}} * \mathrm{D}_{\mathrm{i}, \mathrm{t}} * \mathrm{VLPA}_{\mathrm{i}, \mathrm{t}}+\varepsilon_{\mathrm{i}, \mathrm{t}}
$$

\begin{tabular}{|c|c|c|c|c|}
\hline $\begin{array}{c}\text { Período: } 1996 \text { a } 2006 \\
\text { No de observações: } 99 \\
\text { No de empresas: } 9\end{array}$ & Coeficiente & P-value & Erro Padrão Robusto & Teste $\mathbf{t}$ \\
\hline$\alpha_{1} \mathrm{D}_{\mathrm{i}, \mathrm{t}} * \mathrm{VLPA}_{\mathrm{i}, \mathrm{t}-1}$ & 1,769 & $0,000 * * *$ & 0,180 & 9,83 \\
\hline$\alpha_{2}$ VLPA $_{i, t-1}$ & $-0,269$ & $0,029 * *$ & 0,121 & $-2,22$ \\
\hline$\alpha_{3} \operatorname{VLPA}_{\mathrm{i}, \mathrm{t}-1} * \mathrm{D}_{\mathrm{i}, \mathrm{t}} * \mathrm{VLPA}_{\mathrm{i}, \mathrm{t}}$ & $-0,131$ & $0,006 * *$ & 0,046 & $-2,84$ \\
\hline$\alpha_{4} A_{i, t} * V L P A_{i, t-1}$ & $-0,026$ & 0,848 & 0,136 & $-0,19$ \\
\hline$\alpha_{5} \mathrm{~A}_{\mathrm{i}, \mathrm{t}} * \mathrm{VLPA}_{\mathrm{i}, \mathrm{t}-1} * \mathrm{D}_{\mathrm{i}, \mathrm{t}} * \mathrm{VLPA}_{\mathrm{i}, \mathrm{t}}$ & 0,033 & 0,150 & 0,023 & 1,45 \\
\hline
\end{tabular}

Notas: ***Significância estatística de 1\%; **Significância estatística de 5\%. 
O modelo em análise considera os dois principais papéis dos accruals: i) a mitigação da variabilidade dos fluxos de caixa e ii) o reconhecimento assimétrico de ganhos e perdas não realizados (BALL; SHIVAKUMAR, 2005). Observe-se, nos resultados, que as variáveis "VLPA $A_{i, t-1} "$ e "VLPA $A_{i, t-1} * D_{i, t} * \operatorname{VLPA}_{i, t} "$ apresentaram coeficientes $\alpha_{2}$ e $\alpha_{3}$ significativos, respectivamente, ao nível de significância de 5\%. O resultado do coeficiente $\alpha_{2}$ é significante e menor que zero $\left(\alpha_{2}<0\right)$, demonstrando que os ganhos são um componente transitório do resultado e nos períodos subsequentes tendem a ser revertidos. Essa característica é a esperada em situações que os accruals são utilizados para se reconhecerem, de maneira tempestiva, os ganhos no resultado. Adicionalmente, a soma dos coeficientes $\alpha_{2}+\alpha_{3}<0$ indica que as perdas econômicas são reconhecidas transitoriamente e tendem a reverter, demonstrando mais uma vez a utilização dos accruals como instrumento de reconhecimento tempestivo. No que tange à qualidade do lucro mensurada pelo modelo de BS (2005) a partir de 2002, verifica-se que a variável “ $\mathrm{A}_{\mathrm{i}, \mathrm{t}} * \mathrm{VLPA}_{\mathrm{i}, \mathrm{t}-1} * \mathrm{D}_{\mathrm{i}, \mathrm{t}} * \mathrm{VLPA}_{\mathrm{i}, \mathrm{t}}$ ” não apresentou coeficiente significativo, mesmo ao nível de 10\% de significância. Dessa forma, também, pode-se dizer que não há indícios de que, a partir de 2002 (ano de promulgação da SOX), houve aumento da qualidade do lucro contábil das empresas que emitiram ADR antes de 2002. Esse resultado sugere a rejeição da hipótese do trabalho. Uma das intuições que podem resultar desses indícios é que as empresas com ADRs emitidos antes de 2002 já apuravam seu lucro de maneira a considerar o reconhecimento assimétrico de ganhos e perdas não realizados, de maneira que a SOX não causou influência direta nessa característica do lucro dessas empresas.

Após a análise dos resultados dos dois modelos, Basu (1997) e BS (2005), observe-se que o modelo de Basu (1997) parece conseguir captar indícios de conservadorismo a partir de 2002, ao contrário do Modelo de BS (2005) que não captou alterações no reconhecimento assimétrico de ganhos e perdas não realizados.

Contudo, apesar dos resultados apresentados no modelo de Basu (1997), só se pode afirmar que há indícios de que ocorreu aumento do conservadorismo pela SOX e não por outros motivos econômicos intrínsecos ao período 2002 a 2006, na apuração dos resultados apresentados na seção 6.2, caso a dummy " $\mathrm{ADR}_{\mathrm{i}, \mathrm{t}}$ " seja significativa.

\subsubsection{Análise dos resultados do Cash Flow Model (BALL; SHIVAKUMAR, 2005)}

Apresentam-se na Tabela 5, os resultados das regressões estimadas em painel pelo Cash Flow Model (2005) para as empresas que emitiram ADR antes de 2002. 
Tabela 5 - Resultados da regressão - Amostra só com empresas com ADR - Cash Flow Model (2005)

Equação (5): $\mathrm{ACT}_{\mathrm{i}, \mathrm{t}}=\alpha_{0}+\alpha_{1} \mathrm{FCO}_{\mathrm{i}, \mathrm{t}}+\alpha_{2} \mathrm{~A}_{\mathrm{i}, \mathrm{t}}+\varepsilon_{\mathrm{i}, \mathrm{t}}$

\begin{tabular}{c|c|c|c|c}
\hline $\begin{array}{c}\text { Período: 1996 a 2006 } \\
\mathbf{N}^{\circ} \text { de observações: 99 } \\
\mathbf{N}^{\circ} \text { de empresas: 9 }\end{array}$ & Coeficiente & $\boldsymbol{P}$-value & Erro Padrão Robusto & Teste t \\
\hline$\alpha_{1} \mathrm{FCO}_{\mathrm{i}, \mathrm{t}}$ & $-0,402$ & $0,000^{* * *}$ & 0,063 & $-6,39$ \\
\hline$\alpha_{2} \mathrm{~A}_{\mathrm{i}, \mathrm{t}}$ & 0,007 & 0,369 & 0,007 & 0,90 \\
\hline
\end{tabular}

Notas: ***Significância estatística de $1 \%$.

Observe-se que, nos resultados da regressão estimada em painel, o sinal do coeficiente $\alpha_{1}$ está negativo, assumindo a correlação negativa em função do comportamento dos accruals total, em linha com o trabalho de Ball e Shivakumar (2005).

Contudo, em relação ao gerenciamento de resultados mensurado pelo modelo do Cash Flow Model (2005) a partir de 2002, verifica-se que a variável " $\mathrm{A}_{\mathrm{i}, \mathrm{t}}$ " não apresentou coeficiente significativo, mesmo ao nível de $10 \%$. Dessa forma, pode-se dizer que não há indícios de que, a partir de 2002, as empresas que emitiram ADR antes de 2002 reduziram o nível de accruals discricionários. Esse resultado, igualmente, sugere a rejeição da hipótese do trabalho.

\subsection{Análise dos resultados de todas as empresas brasileiras}

Analisam-se, nos tópicos a seguir, os resultados dos três modelos estimados em painel a partir de amostra que contém todas as empresas brasileiras.

\subsubsection{Análise dos resultados do Modelo de Basu (1997)}

$\mathrm{Na}$ Tabela 6, apresentam-se os resultados das regressões estimadas em painel pelo Modelo de Basu (1997) para todas as empresas que compõem a amostragem final.

Tabela 6 - Resultados da regressão - Amostra com todas empresas brasileiras - Modelo de Basu (1997) Equação (1) - $\operatorname{LPA}_{\mathrm{i}, \mathrm{t}} / \mathrm{P}_{\mathrm{i}, \mathrm{t}}=\alpha_{0}+\alpha_{1} \mathrm{D}_{\mathrm{i}, \mathrm{t}}+\alpha_{2} \mathrm{R}_{\mathrm{i}, \mathrm{t}}+\alpha_{3} \mathrm{D}_{\mathrm{i}, \mathrm{t}} * \mathrm{R}_{\mathrm{i}, \mathrm{t}}+\alpha_{4} \mathrm{~A}_{\mathrm{i}, \mathrm{t}} * \mathrm{D}_{\mathrm{i}, \mathrm{t}} * \mathrm{R}_{\mathrm{i}, \mathrm{t}}+\alpha_{5} \mathrm{ADR}_{\mathrm{i}, \mathrm{t}} * \mathrm{~A}_{\mathrm{i}, \mathrm{t}} * \mathrm{D}_{\mathrm{i}, \mathrm{t}} * \mathrm{R}_{\mathrm{i}, \mathrm{t}}+\varepsilon_{\mathrm{i}, \mathrm{t}}$

\begin{tabular}{c|c|c|c|c}
\hline Período: 1996 a 2006 & Coeficiente & P-value & Erro Padrão Robusto & Teste t \\
\hline$\alpha_{1} \mathrm{D}_{\mathrm{i}, \mathrm{t}}$ & 0,333 & 0,143 & 0,227 & 1,47 \\
\hline$\alpha_{2} \mathrm{R}_{\mathrm{i}, \mathrm{t}}$ & 0,887 & 0,101 & 0,540 & 1,64 \\
\hline$\alpha_{3} \mathrm{D}_{\mathrm{i}, \mathrm{t}} * \mathrm{R}_{\mathrm{i}, \mathrm{t}}$ & $-0,619$ & 0,121 & 0,399 & $-1,55$ \\
\hline$\alpha_{4} \mathrm{~A}_{\mathrm{i}, \mathrm{t}}{ }^{*} \mathrm{D}_{\mathrm{i}, \mathrm{t}} * \mathrm{R}_{\mathrm{i}, \mathrm{t}}$ & 2,331 & $0,054 *$ & 1,208 & 1,93 \\
\hline$\alpha_{5} \mathrm{ADR}_{\mathrm{i}, \mathrm{t}} * \mathrm{~A}_{\mathrm{i}, \mathrm{t}} * \mathrm{D}_{\mathrm{i}, \mathrm{t}} * \mathrm{R}_{\mathrm{i}, \mathrm{t}}$ & $-1,423$ & 0,405 & 0,076 & $-0,83$ \\
\hline
\end{tabular}

Notas: * Significância estatística de $10 \%$. 
Novamente, observe-se que as variáveis " $D_{i, t}$ " e " $D_{i, t} * R_{i, t}$ ", assim como nos resultados apresentados na Tabela 9, não revelaram coeficientes $\alpha_{1}$ e $\alpha_{3}$ significativos, respectivamente, mesmo ao nível de significância de $10 \%$. Assim, não se pode inferir que a informação contábil das empresas brasileiras seja conservadora utilizando-se o modelo de Basu (1997).

No que tange ao impacto a partir de 2002 sobre o conservadorismo de Basu (1997), verifica-se que a variável " $\mathrm{A}_{\mathrm{i}, \mathrm{t}} * \mathrm{D}_{\mathrm{i}, \mathrm{t}} * \mathrm{R}_{\mathrm{i}, \mathrm{t}}$ " apresentou coeficiente significativo ao nível de $5 \%$. Dessa forma, também se pode dizer que há indícios de que a informação contábil de todas as empresas que compõem a amostra final, se tornou mais conservadora a partir de 2002.

Já a variável "ADR $R_{i, t} * A_{i, t} * D_{i, t} * R_{i, t}$ ", que mensura impacto adicional nas empresas que emitem ADR, não apresentou coeficiente significativo mesmo ao nível de $10 \%$. Isso sugere que o indício de aumento no conservadorismo contábil a partir do ano de 2002 talvez não tenha ocorrido pelo impacto da SOX e, sim, por outros motivos econômicos do mercado brasileiro no período que compreende os anos de 2002 a 2006. Esse resultado sugere a rejeição da hipótese do trabalho.

\subsubsection{Análise dos resultados do Modelo adaptado de BS (2005)}

Apresenta-se na Tabela 7 os resultados das regressões estimadas em painel pelo modelo de BS (2005) para todas as empresas que compõem a amostragem final.

Tabela 7 - Resultado da regressão - Amostra com todas empresas brasileiras - Modelo de BS (2005)

Equação (2) - VLPA $A_{i, t}=\alpha_{0}+\alpha_{1} D_{i, t} * V_{L P A} A_{i, t-1}+\alpha_{2} \operatorname{VLPA}_{i, t-1}+\alpha_{3} \operatorname{VLPA}_{\mathrm{i}, \mathrm{t}-1} * \mathrm{D}_{\mathrm{i}, \mathrm{t}} * \operatorname{VLPA}_{\mathrm{i}, \mathrm{t}}+\alpha_{4} \mathrm{~A}_{\mathrm{i}, \mathrm{t}} * \operatorname{VLPA}_{\mathrm{i}, \mathrm{t}-1}$

$$
+\alpha_{5} \mathrm{~A}_{\mathrm{i}, \mathrm{t}} * \mathrm{VLPA}_{\mathrm{i}, \mathrm{t}-1} * \mathrm{D}_{\mathrm{i}, \mathrm{t}} * \mathrm{VLPA}_{\mathrm{i}, \mathrm{t}}+\varepsilon_{\mathrm{i}, \mathrm{t}}
$$

\begin{tabular}{c|c|c|c|c}
\hline $\begin{array}{c}\text { Período: 1996 a 2006 } \\
\mathbf{N}^{\circ} \text { de observações: } 660 \\
\mathbf{N}^{\circ} \text { de empresas: } 60\end{array}$ & Coeficiente & P-value & Erro Padrão Robusto & Teste t \\
\hline$\alpha_{1} \mathrm{D}_{\mathrm{i}, \mathrm{t}} * \mathrm{VLPA}_{\mathrm{i}, \mathrm{t}-1}$ & & & & \\
\hline$\alpha_{2} \mathrm{VLPA}_{\mathrm{i}, \mathrm{t}}$ & 1,400 & $0,000 * * *$ & 0,081 & 17,20 \\
\hline$\alpha_{3} \mathrm{VLPA}_{\mathrm{i}, \mathrm{t}-1} * \mathrm{D}_{\mathrm{i}, \mathrm{t}} * \mathrm{VLPA}_{\mathrm{i}, \mathrm{t}}$ & $-0,169$ & 0,103 & 0,103 & $-1,63$ \\
\hline$\alpha_{4} \mathrm{~A}_{\mathrm{i}, \mathrm{t}} * \mathrm{VLPA}_{\mathrm{i}, \mathrm{t}-1}$ & $-0,049$ & 0,115 & 0,031 & $-1,58$ \\
\hline$\alpha_{5} \mathrm{~A}_{\mathrm{i}, \mathrm{t}} * \mathrm{VLPA}_{\mathrm{i}, \mathrm{t}-1} * \mathrm{D}_{\mathrm{i}, \mathrm{t}} * \mathrm{VLPA}_{\mathrm{i}, \mathrm{t}}$ & 0,021 & 0,473 & 0,157 & 0,73 \\
\hline$\alpha_{6} \mathrm{ADR}_{\mathrm{i}, \mathrm{t}} * \mathrm{~A}_{\mathrm{i}, \mathrm{t}} * \mathrm{VLPA}_{\mathrm{i}, \mathrm{t}-1}$ & $-0,063$ & 0,483 & 0,029 & $-0,70$ \\
\hline$\alpha_{7} \mathrm{ADR}_{\mathrm{i}, \mathrm{t}} * \mathrm{~A}_{\mathrm{i}, \mathrm{t}} * \mathrm{VLPA}_{\mathrm{i}, \mathrm{t}-1} * \mathrm{D}_{\mathrm{i}, \mathrm{t}} * \mathrm{VLPA}_{\mathrm{i}, \mathrm{t}}$ & 0,005 & 0,703 & 0,089 & 0,38 \\
\hline
\end{tabular}

Notas: ***Significância estatística de $1 \%$.

Observe-se, nos resultados, que as variáveis "VLPA $A_{i, t-1}$ " e "VLPA $A_{i, t-1} * D_{i, t} * V_{L P A}$, , ", revelaram coeficientes $\alpha_{2}$ e $\alpha_{3}$ não significativos, respectivamente, mesmo ao nível de 
significância de 10\%. Assim, utilizando-se o modelo de BS (2005) não se pode inferir que a informação contábil das empresas brasileiras não apresente o reconhecimento assimétrico de ganhos e perdas não realizados.

No que tange ao impacto das empresas que emitem ADR sobre o reconhecimento assimétrico de ganhos e perdas não realizados avaliados pelo modelo de BS (2005), verificase que o resultado do coeficiente $\alpha_{6}$ é contrário ao esperado, uma vez que, ao ser menor que zero $\left(\alpha_{2}<0\right)$, demonstra que os ganhos passam a ser um componente transitório do resultado e nos períodos subsequentes tendem a ser revertidos.

Verifica-se que tanto em relação ao impacto a partir do ano de 2002, quanto ao impacto das empresas que emitem ADR, as variáveis " $\mathrm{A}_{\mathrm{i}, \mathrm{t}}$ *VLPA $\mathrm{A}_{\mathrm{i}, \mathrm{t}-\mathrm{l}}$ ", “A $\mathrm{A}_{\mathrm{i}, \mathrm{t}} * \mathrm{VLPA}_{\mathrm{i}, \mathrm{t}-\mathrm{1}} * \mathrm{D}_{\mathrm{i}, \mathrm{t}} * \mathrm{VLPA}_{\mathrm{i}, \mathrm{t}}$ " e “ADR $\mathrm{AD}_{\mathrm{i}, \mathrm{t}} \mathrm{A}_{\mathrm{i}, \mathrm{t}} * \mathrm{VLPA}_{\mathrm{i}, \mathrm{t}-1} * \mathrm{D}_{\mathrm{i}, \mathrm{t}} * \mathrm{VLPA}_{\mathrm{i}, \mathrm{t}}$ " não apresentaram coeficientes $\alpha_{4}, \alpha_{5}$ e $\alpha_{7}$, respectivamente, significativos mesmo ao nível de $10 \%$ de significância.

Dessa forma, também se pode dizer que não há indícios de que a informação contábil das empresas brasileiras passou a ter o reconhecimento assimétrico de ganhos e perdas não realizados diferenciado depois da SOX.

Portanto, não há indícios de que ocorreu aumento da qualidade do lucro contábil depois da promulgação da SOX, também em relação aos resultados apresentados no modelo de BS (1997). Isso sugere a rejeição da hipótese do trabalho.

\subsubsection{Análise dos Resultados do Cash Flow Model (BALL; SHIVAKUMAR, 2005)}

Apresentam-se, na Tabela 8, os resultados da regressão estimada em painel pelo Cash Flow Model (2005) para todas as empresas que compõem a amostragem final.

Tabela 8 - Resultado da regressão - Amostra com todas empresas brasileiras - Cash Flow Model (2005)

$$
\text { Equação (5) - } \mathrm{ACT}_{\mathrm{i}, \mathrm{t}}=\alpha_{0}+\alpha_{1} \mathrm{FCO}_{\mathrm{i}, \mathrm{t}}+\alpha_{2} \mathrm{~A}_{\mathrm{i}, \mathrm{t}}+\alpha_{3} \mathrm{ADR}_{\mathrm{i}, \mathrm{t}} * \mathrm{~A}_{\mathrm{i}, \mathrm{t}}+\varepsilon_{\mathrm{i}, \mathrm{t}}
$$

\begin{tabular}{c|c|c|c|c}
\hline $\begin{array}{c}\text { Período: 1996 a 2006 } \\
\mathbf{N}^{\circ} \text { de observações: 660 } \\
\mathbf{N}^{\circ} \text { de empresas: 60 }\end{array}$ & Coeficiente & $\boldsymbol{P}$-value & Erro Padrão Robusto & Teste t \\
\hline$\alpha_{1} \mathrm{FCO}_{\mathrm{i}, \mathrm{t}}$ & $-0,173$ & $0,006^{* * *}$ & 0,0633 & $-2,73$ \\
\hline$\alpha_{2} \mathrm{~A}_{\mathrm{i}, \mathrm{t}}$ & 0,005 & 0,382 & 0,006 & 0,88 \\
\hline$\alpha_{3} \mathrm{ADR}_{\mathrm{i}, \mathrm{t}} * \mathrm{~A}_{\mathrm{i}, \mathrm{t}}$ & $-0,051$ & 0,534 & 0,008 & $-7,71$ \\
\hline
\end{tabular}

Notas: ***Significância estatística de $1 \%$. 
Observe-se que, nos resultados, o sinal do coeficiente $\alpha_{1}$ está negativo, assumindo a correlação negativa em função do comportamento dos accruals total, em linha com o trabalho de Ball e Shivakumar (2005). Em relação tanto ao impacto a partir do ano de 2002, quanto ao impacto adicional específico nas empresas que emitem ADR, verifica-se que as variáveis “ $\mathrm{A}_{\mathrm{i}, \mathrm{t}}$ " e "ADR $\mathrm{AD}_{\mathrm{i}, \mathrm{t}} \mathrm{A}_{\mathrm{i}, \mathrm{t}}$ " não apresentam coeficientes $\alpha_{2}$ e $\alpha_{3}$ significativos, respectivamente, mesmo ao nível de $10 \%$.

Dessa forma, pode-se dizer que não há indícios de redução do nível de accruals discricionários a partir de 2002, tanto nas empresas com ADR quanto para todas as empresas que compõem a amostra final. Esse resultado sugere a rejeição da hipótese do trabalho.

\section{CONCLUSÃO}

Procurou-se investigar neste trabalho, o impacto da SOX na qualidade do lucro das empresas brasileiras que emitiram ADR antes de 2002. Em suma, analisou-se o conservadorismo, a assimetria de reconhecimento de boas e más notícias e os níveis de gerenciamento de resultado das empresas sujeitas à SOX, antes e após sua promulgação.

Os resultados, utilizando-se os modelos de BS (2005), tanto em relação ao impacto do reconhecimento assimétrico de ganhos e perdas não realizadas a partir de 2002, quanto ao impacto devido à emissão de ADR, não foram significativos, indicando que não há indícios de alteração nessas características do lucro após a implementação da SOX.

Apesar de os resultados em relação ao modelo de Basu (1997) apresentarem indícios de conservadorismo a partir de 2002, em função do coeficiente significativo da dummy “ANO", tanto para as empresas que emitiram ADR antes de 2002, quanto para todas as empresas brasileiras, o termo “ADR*ANO" não apresentou coeficiente significativo mesmo ao nível de $10 \%$. Dessa forma, há indícios de que o aumento no conservadorismo contábil a partir de 2002, talvez não ocorreu pelo impacto da SOX e, sim, por outros motivos econômicos do mercado brasileiro no período de 2002 a 2006.

Tais resultados podem ter sido influenciados pelo fato de o mercado brasileiro ser classificado como code law, ser menos desenvolvido e apresentar controle acionário concentrado.

Contudo, estão em linha com as afirmações de Siegel (2005) de que o arcabouço legal que envolve as ADR não tem influência sobre a administração das empresas estrangeiras, não sendo capaz de aumentar a qualidade do lucro contábil. 
Com base nos resultados, sugere-se a ampliação deste estudo em outros países e a investigação dos efeitos econômicos que motivaram os resultados aqui encontrados.

\section{Referências}

BALL, R. et al. The effect of institutional factors on the properties of accounting earnings. Journal of Accounting and Economics, v. 29, p. 1-51, 2000.

BALL, R.; SHIVAKUMAR, L. Earnings quality in UK private firms. Journal of Accounting and Economics, v. 39, p. 83-128, 2005.

BASU, S. The Conservatism principle and the asymmetric timeliness of earnings. Journal of Accounting and Economics, v. 24, p. 3-37, 1997.

BEAVER, W. H. Conservatism. Working paper, Stanford University, San Francisco, CA, 1993.

COELHO, A. C.; LIMA, I. S. Qualidade informacional e conservadorismo nos resultados contábeis publicados no Brasil. Revista Contabilidade e Finanças, v. 18, p. 38-49, 2007.

; CIA, J. N. S.; LIMA, I. S. Conservadorismo condicional na divulgação de lucros: diferenças entre companhias abertas brasileiras emissoras e não emissoras de ADRs e entre sistemas contábeis. In: CONGRESSO USP DE CONTROLADORIA E CONTABILIDADE, 8., 2008, São Paulo. Anais... São Paulo: USP, 2008.

; LOPES, A. B. Avaliação da prática de apropriação discricionária na apuração de lucro por companhias abertas brasileiras conforme seu grau de alavancagem. In: ENCONTRO DA ASSOCIAÇÃO NACIONAL DE PROGRAMAS DE PÓS-GRADUAÇÃO EM ADMINISTRAÇÃO, 29., 2005, Brasília. Anais... Brasília: ANPAD, 2005.

COFFEE, J. The Future as history: the prospects for global convergence in corporate governance and its implications. Northwestern Law Review, n. 93, p. 641-707, 1999.

COSTA, F. M.; COSTA, A. C. O. Um estudo da aplicação do conservadorismo em cinco países da América do Sul. In: ENCONTRO DA ASSOCIAÇÃO NACIONAL DE PROGRAMAS DE PÓS-GRADUAÇÃO EM ADMINISTRAÇÃO, 29., 2005, Brasília. Anais... Brasília: ANPAD, 2005.

; ALMEIDA, J. C. G.; SCALZER, R. S. Níveis diferenciados de governança corporativa da Bovespa e grau de conservadorismo: estudo empírico em companhias abertas listadas na Bovespa. In: CONGRESSO USP DE CONTROLADORIA E CONTABILIDADE, 6., 2006, São Paulo. Anais... São Paulo: USP, 2006.

DEFOND, M. L.; FRANCIS, J. R. Audit research after Sarbanes-Oxley. Auditing: A Journal of Practice \& Theory, v. 24, Supplement, p. 5-30, 2005.

DOWNES, J.; GOODMAN, J. E. Dicionário de termos financeiros e de investimentos. Tradução: Ana Rocha Tradutores Associados. São Paulo: Nobel, 1993. 
DURTSCHI, C.; EASTON, P. Earnings management? erroneous inferences based on earnings frequency distributions. Journal of Accounting Research, v. 47, p. 1249-1281, 2009.

JAIN, P. K.; REZAEE, Z. The Sarbanes-Oxley act of 2002 and accouting conservatis. 2004. Disponível em: <http://www.ssrn.com/>. Acesso em: 18 nov. 2006.

LOBO, G. J.; ZHOU, J. Did conservatism in financial reporting increase after the SarbanesOxley act? Inicial evidence. Accouting Horizons, v. 20, n. 1, p. 57-73, mar. 2006.

LOPES, A. B. Uma contribuição ao estudo da relevância da informação contábil para o mercado de capitais: o modelo de Ohlson aplicado à Bovespa. 2001. Tese (Doutorado em Controladoria e Contabilidade) - Departamento de Contabilidade e Atuária, Faculdade de Economia, Administração e Contabilidade, Universidade de São Paulo.

LUBBERINK, M.; HUIJGEN, C. A. Cross-listing in US markets and conservatism: does types of listing matter? 2006. Disponível em: <http://www.ssrn.com>. Acesso em: 12 jul. 2006.

MARTINEZ, A. L. "Gerenciamento" dos resultados contábeis: estudo empírico das companhias abertas brasileiras. São Paulo, 2001. Tese (Doutorado em Controladoria e Contabilidade) - Departamento de Contabilidade e Atuária, Faculdade de Economia, Administração e Contabilidade, Universidade de São Paulo.

PAULO, E.; ANTUNES, M. T. P.; FORMIGONI, H. Estudo sobre o conservadorismo nas companhias abertas e fechadas brasileiras. In: EnANPAD, 30., 2006, Salvador. Anais... Curitiba: ANPAD, 2006, CD-ROM.

PALEPU, K. G.; HEALY, P. M. The fall of enron. The Journal of Economic Perspectives, v. 17, n. 2, p. 3-26, 2003.

PENMAN, S. H.; ZHANG, Xiao-Jun. Accounting conservatism, the quality of earnings, and stock returns. The Accounting Review, v. 77, p. 237-26, 2002.

POPE, P., WALKER, M. International differences in the timeliness, conservatism, and classification of earnings. Journal of Accounting Research, v. 37, p. 53-87, 1999.

SANTOS, L. S. R. Conservadorismo contábil e timeliness: evidências empíricas nos demonstrativos contábeis em US GAAP e BR GAAP das empresas brasileiras com ADRS negociadas na Bolsa de Nova Iorque. 2006. Dissertação (Mestrado em Contabilidade) Programa de Pós-Graduação em Ciências Contábeis da FUCAPE, Vitória.

SARLO NETO, A. et al. O impacto da regulamentação sobre a relação entre o lucro e retorno das ações das empresas dos setores elétrico e financeiro no Brasil. In: ENCONTRO DA ASSOCIAÇÃO NACIONAL DE PROGRAMAS DE PÓS-GRADUAÇÃO EM ADMINISTRAÇÃO, 26., 2002, Salvador. Anais... Salvador: ANPAD, 2002.

SIEGEL, J. Can foreign firms bond themselves effectively by renting U.S. securities laws? Journal of Financial Economics, v. 75, p. 319-359, 2005. 
WATTS, R. L. Conservatism in accounting part I: explanations and implications. Accounting Horizons, v. 17, n. 3, p. 207-221, 2003. Disponível em: <http://proquest.com/>. Acesso em: 27 nov. 2006.

.Conservatism in accounting part II: evidence and research opportunities. Accounting Horizons, v. 17, n. 4, p. 287-301, 2003a. Disponível em: <http://proquest.com/>. Acesso em: 27 nov. 2006. 